\title{
SUCCESSIVE APPROXIMATION METHOD FOR THE CALCULATION OF BENT-COMPRESSED BEAMS OF CONSTANT STIFFNESS
}

\author{
Moussa Sali ${ }^{1}$, V. V. Filatov ${ }^{2}$ and Njomoue Pandong Achille ${ }^{3}$ \\ ${ }^{1,3}$ University of Douala, Faculty of Industrial Engineering, laboratory of Energy, Materials, Modelling and \\ Method (E3M), \\ Po. Box 2071 Douala, Cameroon \\ ${ }^{2}$ Moscow State University of Civil Engineering, Laboratory of Structural Mechanic, Russian Federation
}

\begin{abstract}
The finite difference equations of the successive approximation method (SAM) which substitute the differential equations of bending-compressed of constant stiffness are obtained. Difference equations of SAM, which approximate the limit conditions of the hand ends of the beam, are also obtained: simply supported hand end; rigidly fixed hand end and freehand end. On the basis of the obtained equations, a numerical algorithm was developed for calculating beams of constant thickness under the action of various static loads. According to this algorithm, a program for calculating beams on a computer was performed. The examples presented here show the accuracy of the results and the simplicity of the algorithm. Checks for integral equilibrium conditions of beams were performed to validate the newly obtained results.
\end{abstract}

Key words: Successive Approximation Method, Bent-Compressed Beam, Constant Thickness, Numerical Algorithm, Equilibrium Conditions

\section{INTRODUCTION}

The foundation beams, the columns of some buildings, the pillars of electrical transportation and the chimneys are generally beams of constant or variable stiffness. This type of structure is also found in aeronautical or naval constructions. During the process of their exploitation, these structures undergo the action not only of static charges, but also dynamic loads. The calculation of such structures must be accurate and easy to execute. Despite the practical importance of such elements found in a number of books, many of the questions related to their calculation are still relevant. Many works of researchers address the problem of beams [1-5]. The calculation of these structures requires the implementation of tools for modeling mechanical behavior increasingly sophisticated, and taking into consideration the specificities of these structures. Their calculation by the analytical methods remains very tedious and bulky see for example [1], [3], [6-7]. The numerical methods described in references [4], [5], [8-10] are more efficient. Among numerical methods, the finite element method is the most used, but it presents a number of difficulties such as the formation of the stiffness matrix and the tightening of the mesh around the specific zones. New numerical methods which are much more resonant, simple and that yield appreciable results are being developed by other researchers [2], [4], [11-14].. Among these methods we have the Successive Approximation Method (SAM) which is the subject of this article. More precisely, we will use this method to develop an algorithm for the calculation of bent-compressed beam of constant stiffness. The work is organized as follows. After the introduction that poses the problematic of the subject, we will unfold the methodology of the implementation of the Successive Approximation Method which is declined in three points. We will begin by describing the equations of the model problem, including the boundary conditions, and then introduce new dimensionless parameters into the system of equations thus obtained and into the equations describing the boundary conditions. We will continue by substituting for the new differential equations the finite difference equations of the Successive Approximation Method. This will make it possible to obtain a system of algebraic equations. Finally we will develop a calculation algorithm. Before concluding, we will devote the last part of the work to the validation of our approach (the Successive Approximation Method) through the numerical resolution of the test problems. 


\section{EQUATION OF PROBLEM'S MODEL}

The differential equation of a bending - compression beam of constant stiffness can be obtained as a special case of the differential equation of a flexural - compression plate. For this, it suffices in (2.17) [1] all the partial derivatives with respect to $y$ are equal to zero and the rigidity $D$ replaced by the bending rigidity $E I$ :

$\frac{d^{4} W}{d x^{4}}=\frac{1}{E I}\left(q+N \frac{d^{2} W}{d x^{2}}\right)$

where $\mathrm{W}$ is the deflection of the beam, $\mathrm{q}$ is the intensity of the distributed charge, $\mathrm{N}$ is the normal effort.

Let's put (1) in the form of a system of two second-order differential equations. For that, let's introduce the following expression:

$$
\text { EI } \frac{d^{2} w}{d x^{2}}=-M
$$

In this case (1) will be written as follows:

$\frac{d^{2} M}{d x^{2}}=-\left(q+N \frac{d^{2} w}{d x^{2}}\right)$

When we introduce (2) in (3), we obtain (1). This allows the verification of the transformation performed. Now let's write (3) taking into consideration (2):

$\frac{\mathbf{d}^{2} \mathbf{M}}{\mathbf{d x}^{2}}=-\left(\mathbf{q}-\mathbf{N} \cdot \frac{\mathbf{M}}{\mathrm{EI}}\right)$

$\mathbf{N}$ is considered positive when we have compression. In this case (4) is written by changing the sign of $\mathbf{N}$ :

$\frac{\mathbf{d}^{2} \mathbf{M}}{\mathbf{d x}^{2}}=-\left(\mathbf{q}+\mathbf{N} \cdot \frac{\mathbf{M}}{\mathrm{EI}}\right)$

Thus, instead of (1), we obtain (5) and (2).

\section{METODOLOGY OF THE IMPLEMENTATION OF THE SUCCESSIVE APPROXIMATION METHOD (SAM)}

\subsection{Introduction of New Dimensionless Parameters}

Let us write (5) and (2) as functions of dimensionless parameters. Switching to dimensionless parameters solves not only a single problem, but a whole series of problems.

Thus, from (5), (2), it follows:

$$
\begin{aligned}
& \frac{d^{2} \mathbf{m}}{d \xi^{2}}=-(\mathbf{p}+\mathbf{k} \cdot \mathbf{m}) \\
& \frac{\mathbf{d}^{2} \omega}{d \xi^{2}}=-\mathbf{m}
\end{aligned}
$$

where $\xi=\frac{X}{l} \quad ; \quad m=\frac{M}{q_{0} l^{2}} \quad ; \quad \omega=\frac{W . E I}{q_{0} l^{4}} \quad ; \quad \mathbf{p}=\frac{\mathbf{q}(\mathrm{x})}{q_{0}} ; \quad \mathbf{k}=\frac{N . l^{2}}{E I}$

$l$ is the span of the beam; $q_{0}$ is the intensity of the distributed charge $\mathrm{q}(\mathrm{x})$ is the intensity of a fixed charge.

When we put (8) in (6) and (7), we obtain (5) and (2).

From (8), we notice that by solving (6) and (7), for the fixed values of $P$ and $k$ with respect to $m$ and $w$, we obtain the results for any value of $q_{0}, l$ and $E I$.

The differential equations (6), (7) are resolved taking into consideration the following boundary conditions:

Articulated support: $m=m_{0} ; w=w_{0}$.

Recessed support: $w=w_{0} ; w^{\prime}=w_{0}^{\prime}$.

Free edge: $m=m_{0} ; m^{\prime}=m_{0}^{\prime}+k w^{\prime}$.

In (9) - (11): $w^{\prime}=\frac{\partial w}{\partial \xi} ; m^{\prime}=\frac{\partial m}{\partial \xi}$ are dimensionless derivatives of $W$ and $M$ respectively; $T=\frac{\partial M}{\partial X}=m^{\prime} q_{0} l$ is the shear force; $m_{0}, w_{0}, m_{0}^{\prime}, w_{0}^{\prime}$ are the values of $m, w, m^{\prime}, w^{\prime}$ respectively. In some particular cases those values are equal to zero. In the second equality of (11), the component $k w^{\prime}$ represents the projection of $\bar{q}$, compression load of $k$ on the end of the deformed axis of the beam.

From Figure 1: $\bar{q}=k \sin \alpha$; since the angle is too small, $\sin \alpha=\tan \alpha=w^{\prime}$; in this case $\bar{q}=k \cdot w^{\prime}$; the projection of shear force $m_{0}^{\prime} \cos \alpha=m_{0}^{\prime}$ because $\cos \alpha=1$. 


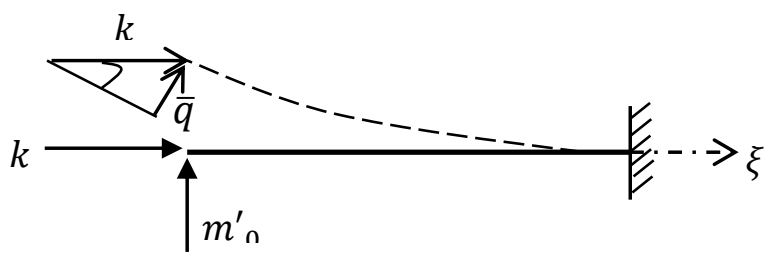

Figure 1. Representation of the components of the force $k$

To build the numerical algorithm of computation, (6) and (7) must be replaced by the finite difference equations of the successive approximations method (SAM).

\subsection{Substitution of the Differential Dimensionless Equations by the Finite Difference Equations of Successive Approximation Method}

In [11], the differential equation of second order......... $\frac{\mathbf{d}^{2} \mathbf{w}}{\mathbf{d} \xi^{2}}=-\mathbf{p}$

Can be substituted for the nod $i$ of a regular mesh with a step $h$ see Figure 2 by the finite difference equation of SAM:

$$
\begin{aligned}
& \quad{ }^{d} w_{i-1}-2{ }^{g} w_{i}+{ }^{g} w_{i+1}+\Delta w_{i}+h \Delta w_{i}^{\prime}=-\frac{h^{2}}{12}\left({ }^{d} p_{i-1}+10{ }^{g} p_{i}+{ }^{g} p_{i+1}\right)+\frac{5 h^{2}}{12} \Delta p_{i}+\frac{h^{3}}{12} \Delta P_{i}^{\prime} \\
& \text { where } \quad w^{\prime}=\frac{d w}{d \xi} ; \quad P^{\prime}=\frac{d P}{d \xi} ; \Delta w_{i}={ }^{g} w_{i}-{ }^{d} w_{i} ; \Delta P_{i}={ }^{g} P_{i}-{ }^{d} P_{i} ; \\
& \Delta w_{i}^{\prime}={ }^{g} w_{i}^{\prime}-{ }^{d} w_{i}^{\prime} ; \Delta P_{i}^{\prime}={ }^{g} P_{i}^{\prime}-{ }^{d} P_{i}^{\prime}
\end{aligned}
$$

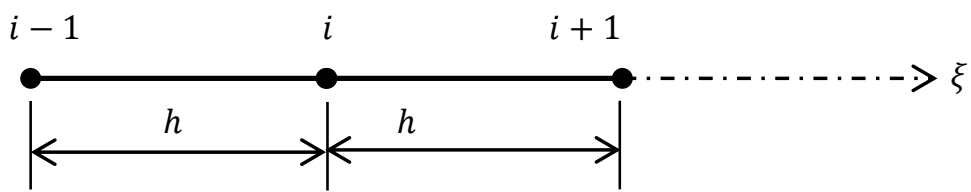

Figure 2. regular mesh

Since in the deduction (13), it has been assumed in [2] that the functions $\mathrm{w}, \mathrm{p}$ and their first derivatives can be discontinuous at certain nods of the mesh. The values of $w, p, w^{\prime}, p^{\prime}$ have left upper indices ' $\mathrm{g}$ ' and'd'. These indices indicate that they are the values of $w, p, w^{\prime}, p^{\prime}$ in the neighborhood of the nod $i$ respectively on the left and on the right.

On the hand-end nods of the beam, if we denote it by $i$, then (1.12) can be substituted by the finite difference equation of SAM:

$$
h w_{i}^{\prime}+w_{i}-{ }^{g} w_{i+1}=\frac{h^{3}}{12} P_{i}^{\prime}+\frac{h^{2}}{12}\left(5 P_{i}+{ }^{g} P_{i+1}\right)
$$

From the comparison of (12) and (6), it follows that to substitute (6), it suffices in (13), (14) $w$ and $P$ to replace respectively by $m$ and $P+k m$ :

$$
\begin{aligned}
& { }^{d} m_{i-1}-2{ }^{g} m_{i}+{ }^{g} m_{i+1}+\Delta m_{i}+h \Delta m_{i}^{\prime}=-\frac{h^{2}}{12}\left[{ }^{d}(P+k m)_{i-1}+10{ }^{g}(P+k m)_{i}+{ }^{g}(P+k m)_{i+1}\right]+ \\
& +\frac{5 h^{2}}{12} \Delta(P+k m)_{i}+\frac{h^{3}}{12} \Delta(P+k m)_{i}^{\prime} \\
& h m_{i}^{\prime}+m_{i}-{ }^{g} m_{i+1}=\frac{h^{3}}{12}(P+k m)_{i}^{\prime}+\frac{h^{2}}{12}\left[5(P+k m)_{i}+{ }^{g}(P+k m)_{i+1}\right]
\end{aligned}
$$

Transforms (15), (16), assuming that only $m$ and $P$ are variable and discontinuous, $k$ is constant. This numerical approach makes it possible to take into consideration the discontinuity of $k$, as well as the calculation of the beams of variable rigidity laying or not on flexible foundations.

Here we will limit ourselves to the beams of constant rigidity not laying on a flexible foundation.

From (15) and (16) we obtain:

$$
\begin{gathered}
\left(1+\frac{h^{2}}{12} k\right){ }^{d} m_{i-1}-2\left(1-\frac{5 h^{2}}{12} k\right){ }^{g} m_{i}+\left(1+\frac{h^{2}}{12} k\right){ }^{g} m_{i+1}+\left(1-\frac{5 h^{2}}{12} k\right) \Delta m_{i}+h\left(1-\frac{h^{2}}{12} k\right) \Delta m_{i}^{\prime}=-\frac{h^{2}}{12}\left({ }^{d} P_{i-1}+\right. \\
\left.10{ }^{g} P_{i}+{ }^{g} P_{i+1}\right)+\frac{5 h^{2}}{12} \Delta P_{i}+\frac{h^{3}}{12} \Delta P_{i}^{\prime}
\end{gathered}
$$


$h\left(1-\frac{h^{2}}{12} k\right) m_{i}^{\prime}+\left(1-\frac{5 h^{2}}{12} k\right) m_{i}-\left(1+\frac{h^{2}}{12} k\right){ }^{g} m_{i+1}=\frac{h^{3}}{12} P_{i}^{\prime}+\frac{h^{2}}{12}\left(5 P_{i}+{ }^{g} P_{i+1}\right)$

Comparing (6) and (7), it follows that to substitute (7), simply replace in (7) and (18) $m, P$ respectively by $w, m$ and put $k=0$. In this case, we consider that $\Delta \mathrm{w}=\Delta \mathrm{w}^{\prime}=0$. After this we get:

$$
\begin{gathered}
w_{i-1}-2 w_{i}+w_{i+1}=-\frac{h^{2}}{12}\left({ }^{d} m_{i-1}+10{ }^{g} m_{i}+{ }^{g} m_{i+1}\right)+\frac{5 h^{2}}{12} \Delta m_{i}+\frac{h^{3}}{12} \Delta m_{i}^{\prime} \\
h w_{i}^{\prime}+w_{i}-w_{i+1}=\frac{h^{3}}{12} m_{i}^{\prime}+\frac{h^{2}}{12}\left(5 m_{i}+{ }^{g} m_{i+1}\right)
\end{gathered}
$$

The equations (17) to (20) when $k=0$ correspond to those obtained in [2] for the calculation of the bending beams if in these last equations one introduces the coefficient $\gamma=1$ which takes into consideration the variation of rigidity. Equations (18), (20) are written for the nod $i$ of the left hand-end of the beam. For the right hand-end of the beam, these equations can be written in substitutes $i+1, g, d$ respectively by $i-1, d, g$ and $\mathrm{m}^{\prime}, \mathrm{w}^{\prime}, \mathrm{p}^{\prime}$ change their sign:

$$
\begin{gathered}
-\left(1+\frac{h^{2}}{12} k\right) m_{i-1}+\left(1-\frac{5 h^{2}}{12} k\right) m_{i}-h\left(1-\frac{h^{2}}{12} k\right) m_{i}^{\prime}=\frac{h^{2}}{12}\left({ }^{d} P_{i-1}+5 P_{i}\right)-\frac{h^{3}}{12} P_{i}^{\prime} \\
-w_{i-1}+w_{i}-h w_{i}^{\prime}=-\frac{h^{3}}{12} m_{i}^{\prime}+\frac{h^{2}}{12}\left({ }^{d} m_{i-1}+5 m_{i}\right)
\end{gathered}
$$

\subsection{Implementation of an Algorithm of the Calculation}

The algorithm for calculating on a regular mesh is as follows. For all the points of the mesh located inside the domain of integration one writes (17), (19) taking into consideration of boundary conditions. For a beam with two articulated ends, these equations are solved simultaneously by considering the boundary conditions (9), where $m=w=0$ at the ends. In the other cases of the limit conditions one associates with (17), (19) either (18), (11) for a free end or (19), (10) for a recess end. The resolution of the equations thus obtained makes it possible to determine $m$ and $w$. From the formula (18) one can determine values of $M$ and $W$ and the shear force will be calculated by following formula: $=m^{\prime} q_{0} l$. Thus digital resolution gives complete results. All parameters of the stress state of the beam are determined.

\section{NUMERICAL VALIDATION OF THE THEORY}

As a first exercise, consider a beam shown in the Figure 3. The dimensionless span of the beam is equal to $1 ; P=k=1$; in addition $k<k_{c r}=\frac{\Pi^{2}}{4} ; P^{\prime}=0$.

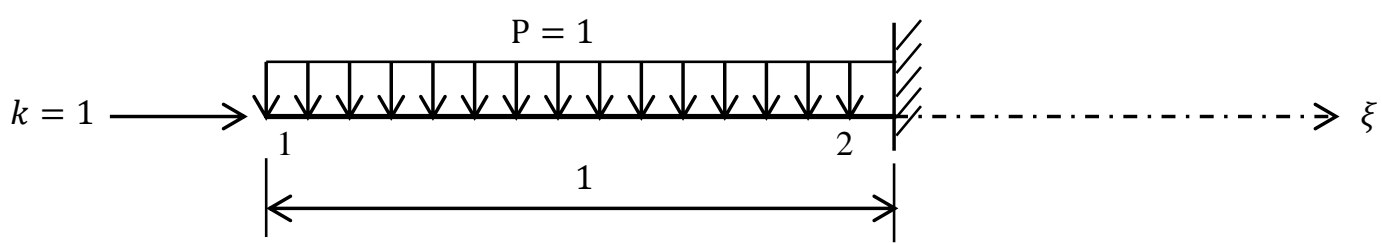

Figure 3. Compressed cantilever beam uniformly loaded throughout its length

The method of successive approximations makes it possible to obtain solutions with a very high precision even with a curse mesh.

Here we will take $h=1$; the nods for which the equations will be written are the nods 1 and 2 which coincide with the hand-ends of the beam:

Boundary conditions when $\xi=0$, according to (11) $m_{1}=0, m_{1}^{\prime}=1 . w_{1}^{\prime}$;

for $\xi=1 \quad w_{2}=0, w_{2}^{\prime}=0$

The unknowns are $m_{2}, w_{1}, w_{1}^{\prime}$. For nod 1, write (18), (20) taking into consideration the boundary conditions and $h=1 ; P=1$ :
a) $1 \cdot\left(1-\frac{1^{2}}{12} \cdot 1\right) w_{1}^{\prime}-\left(1+\frac{1^{2}}{12} \cdot 1\right) m_{2}=\frac{1^{2}}{12}(5 \cdot 1+1)$;
b) $1 . w_{1}^{\prime}+w_{1}=\frac{1}{12} w_{1}^{\prime}+\frac{1}{12} m_{2}$.

For nod 2 write equations (21), (22):

a) $\left(1-\frac{5}{12}\right) m_{2}-\left(1-\frac{1}{12}\right) m_{2}^{\prime}=\frac{1}{12} \cdot 6 ; \quad$ b) $\quad-w_{1}=-\frac{1}{12} m_{2}^{\prime}+\frac{1}{12} \cdot 5 m_{2}$.

By solving (23), (24) with respect to $m_{2}, m_{2}^{\prime}, w_{1}, w_{1}^{\prime}$, we obtain in particular $m_{2}=-0,7143$. The exact value of $m_{2}$ obtained in [3] is $m_{2}=-0,7066$. As a result, the difference is $1.1 \%$.

How to obtain if necessary the improvement of the result? For that, it is enough to increase the number of meshes that is to say to use a mesh more tightened for the computation. We can introduce a nod in the middle of the beam except the nods at the 
ends see Figure 3; at this time $h=\frac{1}{2}$. Equations (18), (20)-(22) should be written considering $h=\frac{1}{2}$. And at the same time, for the nod at the middle of the beam, one must write (17) and (19). These equations will not be presented here, since we will consider a second example in the following for $h=\frac{1}{2}$, but with a different load case see Figure 4.

We denote only that the results obtained when $h=\frac{1}{2} ; h=\frac{1}{4} ; h=\frac{1}{8}$ for the point at the recess are respectively $\mathrm{m}_{2}=$ $-0,7070 ; \mathrm{m}_{2}=-0,7066$. It can be noted that with the increase in the number of meshes, the numerical solution converges towards the exact value.

As a second example, consider the same beam as in the previous example, but with a concentrated force $\Delta m_{2}^{\prime}=1$ in the middle. Figure 4 shows a minimum mesh for this type of loading.

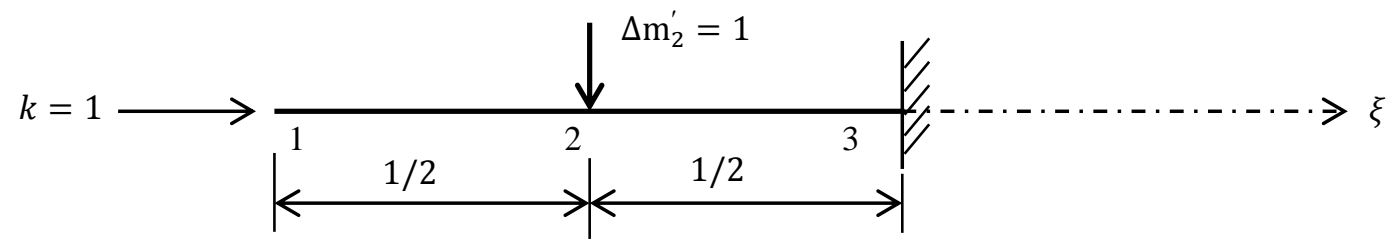

Figure 4. Compressed cantilever beam loaded in the middle by a concentrated force

The boundary conditions are the same as in the previous example. For nods 1 and 3, write (18), (20) to (22) taking into consideration $h=\frac{1}{2} ; P=P^{\prime}=0 ; m_{1}^{\prime}=w_{1}^{\prime}$ :

$$
\begin{aligned}
& \frac{1}{2}\left[1-\frac{1}{12} \cdot \frac{1}{2^{2}}\right] w_{1}^{\prime}-\left(1-\frac{1}{12} \cdot \frac{1}{2^{2}}\right) m_{2}=0 ; \quad \frac{1}{2} \cdot w_{1}^{\prime}+w_{1}-w_{2}=\frac{1}{12} \cdot \frac{1}{2^{3}} w_{1}^{\prime}+\frac{1}{12} \cdot \frac{1}{2^{2}} \cdot m_{2} ; \\
& -\left(1+\frac{1}{12} \cdot \frac{1}{2^{2}}\right) m_{2}+\left(1-\frac{5}{12} \cdot \frac{1}{2^{2}}\right) m_{3}-\frac{1}{2}\left(1-\frac{1}{12} \cdot \frac{1}{2^{2}}\right) m_{3}^{\prime}=0 ; \quad-w_{2}=-\frac{1}{12} \cdot \frac{1}{2^{3}} m_{3}^{\prime}+\frac{1}{12} \cdot \frac{1}{2^{2}}\left(m_{2}+5 m_{3}\right) .
\end{aligned}
$$

For the nod 2 we write (17), (19) while $\Delta m=0$ :

$$
-2\left(1-\frac{5}{12} \cdot \frac{1}{2^{2}}\right) m_{2}+\left(1+\frac{1}{12} \cdot \frac{1}{2^{2}}\right) m_{3}+\frac{1}{2}\left(1-\frac{1}{12} \cdot \frac{1}{2^{2}}\right) \cdot 1=0 ; w_{1}-2 w_{2}=-\frac{1}{12} \cdot \frac{1}{2^{2}}\left(10 m_{2}+m_{3}\right)+\frac{1}{12} \cdot \frac{1}{2^{3}} \cdot 1 .
$$

We thus obtain (25), (26) with six unknowns. By solving this system of equations we obtain in particular: $m_{3}=$ $-0,6704 ; m_{3}^{\prime}=-1,001$.

By increasing the number of meshes twice, we improve the result using the computer: For $h=\frac{1}{4} ; h=\frac{1}{8}$, we obtain for $m_{3}$ the following values $-0,6701 ;-0,6700$ respectively.

A question may arise, how to evaluate the error, if exact solution is not known. The practical question of solving this type of problems, that is to say the numerical algorithm, cannot be useful. The calculation error can be appreciated by studying the static balance of the beam. The resultant of the external load is 1 in this case. According to the rules of the Resistance of Materials, the shear force $m^{\prime}$ at point 3 is equal to 1.001 when $h=\frac{1}{2}$, and is directed upwards. The projection of the horizontal force $\mathrm{k}=1$ is equal to zero. Therefore the balance of the beam is satisfied with an error of $0.1 \%$.

The two previous examples made it possible to show the effectiveness of the use of the boundary conditions for the free and embedded ends, thus the influence of a concentrated transverse load which leads to a discontinuity of $m^{\prime}$ at nod 2 .

The third example will be devoted to the calculation of a beam placed on two articulated supports subjected to a uniformly distributed load on its half, a normal load of traction see Figure 5. This example makes it possible to highlight the discontinuity of the loads and the boundary conditions for the articulated supports.

The boundary conditions are: $m_{1}=m_{3}=w_{1}=w_{3}=0$

According to the data: $P_{3}=0 ; P_{1}=P_{2}=1 ; \Delta P_{2}=1 ; P^{\prime}=0 ; \Delta m^{\prime}=0 ; \mathrm{k}=-1$

$$
\Delta m=0 ; \mathrm{h}=\frac{1}{2} \text {. }
$$

Let's write (17), (19) for the point 2 :

$$
-2\left(1+\frac{5}{12} \cdot \frac{1}{2^{2}}\right) m_{2}=-\frac{1}{12} \cdot \frac{1}{2^{3}}(1+10 \cdot 1)+\frac{1}{12} \cdot \frac{1}{2^{2}} \cdot 1 ; \quad-2 w_{2}=-\frac{1}{12} \cdot \frac{1}{2^{2}} \cdot 10 m_{2} .
$$

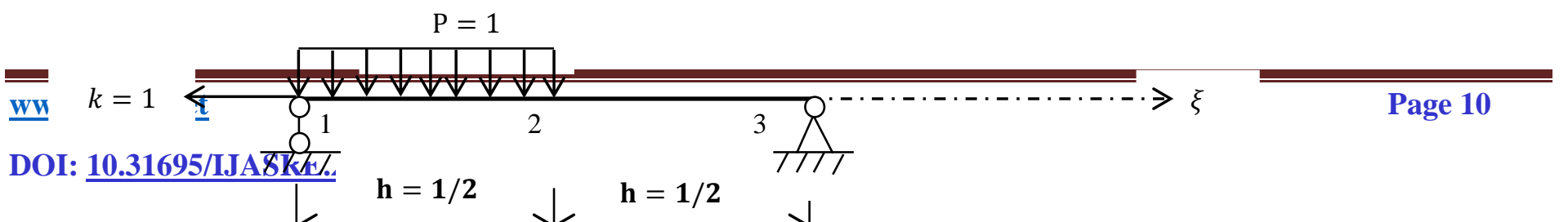


We see that for articulated supports, (27) can be solved in a grouped way; that is, we determine $m$ first, then we determine $w$. For the present case, we obtain: $m_{2}=0,05660 ; w_{2}=0,005896$. In the case of a complete loading of the beam, $P=1$ and (17) must be written as follows:

$$
-2\left(1+\frac{5}{12} \cdot \frac{1}{2^{2}}\right) m_{2}=-\frac{1}{12} \cdot \frac{1}{2^{2}}(1+10.1+1) .
$$

From (28), we obtain $\mathrm{m}_{2}=0,1132$, which is twice as large as the value obtained for half loading. This value is practically equal to the exact value that is $\mathrm{m}_{2}=0,1131$ obtained in [4].

\section{COCLUSION}

At the end of this work we succeeded in transforming the fourth-order differential equation into a system of two differential equations. Then we replaced the parameters of the system equations, as well as those of the boundary conditions by dimensionless parameters. The equations thus obtained have been substituted by the finite difference equations of the Successive Approximation method. This allowed us to obtain systems of algebraic equations whose resolution taking into consideration the boundary conditions was carried out using the iterative Gauss-Seidel method. This allowed us to avoid the preliminary formulation of matrix with unknowns. The algorithm developed here is simple and made it possible to solve the calculation problems of tri constant thickness isotropic thin beams subjected to flexion combined with compression. Different combinations of the most commonly encountered boundary conditions were examined with different type of loads. The results obtained in the various examples show good convergence. Which shows the stability of the method.

\section{REFERENCES}

1. S. P. Timoshenko, Theory of elasticity (Ed. NAHUKOVA DUMKA, Kiev, 1972) 501 p.

2. R. F. Gabassov, A. R. Gabassov, V. V. Filatov. Numerical construction of discontinuous solutions to the problems of constructive mechanics (Ed. ABC. Moscow, 2008) p.277.

3. G. S. Vardanian, V. I. Andreev, N. M. Atapov, A. A. Gorshkov. Material resistance with theory of elasticity and plasticity (Ed. ASB, M., 1995) p568.

4. Structural ship mechanics and elasticity theory (Ed. V.A. Postnov. T.2 - L: Shipbuildingь 1987) p416.

5. R. F. Gabbassov, numerical solution of problems of construction mechanics with discontinuous parameters, doctoral diss., PhysMaths Institute of Moscow., 1989.

6. P. M. Varbak. Development and application of the method of nets to the calculation of plates (Ed. Institute of Building Mechanics part I 1949) p136.

7. E. B. Koreneva. Analytical methods for calculating variable thickness plates and their practical applications (ASB, M., 2009) p238.

8. C. A. Ivanov. Analysis of bent plates by the finite element method. Ed Marsi. 1972, (4) p. 25-31.

9. A. V. Alexandrov. Numerical solution of linear differential equations by means of the differentiation matrix. Ed. MIIT, Moscow, 1961, (131), p253-266.

10. Moussa Sali, The calculation of bending beams and slabs of variable rigidity subject to the dynamic loads, diss. of Ph.D., Moscow State University of Civil Engineering, Moscow 2002.

11. Moussa Sali, Lontsi Frédéric, Oumarou Hamandjoda, Danwé Raidandi, “Calculation of Plates on Elastic Foundation by the Generalized Equations of Finite Difference Method', The International Journal of Engineering and Science IJES, 2018, Vol. (7), Issue 8 Ver.I, p. 32-38. 
12. V. V. Filatov, S Moussa. “About the Assessment of Deformability of Cross Braces when Designing Composite Plates according to the A. A. Rzhanitsyn's Theory', Ed. Industrial and Civil Engineering. 2010, (2), p. 28 - 29.

13. V. V. Filatov, The calculation of compressed-curved beams and slabs on a non-continuous elastic base, diss. of Ph.D., Moscow State University of Civil Engineering, Moscow 1999.

14. Moussa Sali, Amba Jean Chills, "The Finite Difference Equations of Successive Approximation Method for the Calculation of Bending Beams of Variable Thickness under the Static Action of Loads', The International Journal of Advances in Scientific Research and Engineering, IJASRE, 2019, Vol.5, Issue 4, pp. 240-248. 